\title{
Positive-Contrast Cellular MR Imaging Using Susceptibility Weighted Echo-time Encoding Technique (SWEET)
}

\author{
Young Beom Kim ${ }^{1}$, Ki Hyun Bae ${ }^{2}$, Yuhan Lee², Tae Gwan Park², Seung-Schik Yoo ${ }^{3}$ and HyunWook \\ Park $^{1}$ \\ ${ }^{1}$ Department of Electrical Engineering, Korea Advanced Institute of Science and Technology (KAIST), Daejeon, Korea \\ ${ }^{2}$ Department of Biological Science, Korea Advanced Institute of Science and Technology (KAIST), Daejeon, Korea \\ ${ }^{3}$ Department of Radiology, Brigham and Women’s Hospital, Harvard Medical School, Boston, USA
}

\begin{abstract}
The MRI (Magnetic Resonance Imaging) detection and tracking of superparamagnetic iron-oxide particle (SPIO)-labeled cells is expected to play an important role in cell-replacement therapies. SPIO-loaded cells reduce MR signal caused by dephasing due to the magnetic field inhomogeneity near the cells. This signal reduction is typically used for detecting/tracking the labeled cells when it is contrasted to the surrounding tissues (i.e. negative-contrast). However, the negative-contrast can be confused with the signal voids caused by other sources such as tissue inhomogeneity or partial volume effect. Therefore, selective and positive-contrast imaging for SPIO-labeled cells is warranted with the suppression of signals from background tissues. We were motivated to investigate a simple method to detect SPIO-labeled cells with positive-contrast by using susceptibility weighted echo-time encoding technique (SWEET). Human cervix carcinoma cells (KB) were labeled with dextran-coated iron-oxide particles (Feridex), and placed in the gel phantom. Consequently, the difference between the conventional spin-echo images and the echo-shifted images using SWEET rendered the positive signal manifestation from the labeled-cells. Gradient-echo sequence was used to confirm the location of labeled cells. We demonstrated that the method can be readily implemented without the use of dedicated pulse sequence programming. The method can separate out the magnetic susceptibility effect of SPIOloaded cells from the main field-inhomogeneity and chemicalshift artifact.
\end{abstract}

Keywords- cellular MRI, positive-contrast, SWEET, superparamagnetic iron-oxide particle, $\mathrm{KB}$ cell

\section{INTRODUCTION}

With the introduction of cell-replacement therapies [1], there are growing interest to (1) visualize cell migration, (2) to track transplanted cells, and (3) to monitor cell populations noninvasively, repeatedly, and in vivo. Recently a number of imaging modalities have been presented for cell imaging such as optical imaging, positron emission tomography, and magnetic resonance imaging (MRI). Among them, due to its capability of resolving soft tissue anatomies in three dimensions with high spatial resolution, MRI is gaining popularity to image/track cells that are labeled with superparamagnetic contrast agents. Unlike PET, the MRI does not require the radioactive tagging agents, thus allows for repeated measurements. The effective duration of the SPIO-labeling is more than 6 weeks. This capability is especially important in homing and monitoring the migration or the proliferation of cells during cell-replacement therapies.

One of the techniques required for the MRI detection of group/individual cells is labeling cells with superparamagnetic iron-oxide particle(s) (SPIO). SPIO-loaded cells cause a local magnetic field inhomogeneity near the cells, thereby reducing MR signal associated with dephasing. Therefore, the cell(s) is visualized as signal voids contrasted to a brighter background (called negative-contrast). This negative-contrast has been typically used for the detection of the SPIO-labeled cells; however, the negative-contrast has a typical drawback of being confused with the signal voids caused by other objects in the image - e.g. tissue inhomogeneity or partial volume effect. Generally, positivecontrast is desirable for the feature detection; therefore, positive-contrast for SPIO-labeled cells is warranted with suppression of signals from the background tissues.

Other investigators have proposed several methods to elicit positive-contrast in MR imaging of SPIO-loaded cells using spectrally selective method [2, 3]. For example, Inversion-Recovery ON-resonance water suppression (IRON) method used a spectrally-selective saturation pre-RF pulse to suppress the signal originating from on-resonant protons in the background tissue while preserving the signal from off-resonant spins in proximity to the SPIO particles. However, since the size of the signal-enhanced region is dependent on the bandwidth of the water suppression pulse, this scheme requires extra steps to adjust the center frequency and bandwidth of the pre-pulse to locate the exact site proximal to the cells.

Cunningham and colleagues presented a positive-contrast imaging method using frequency-selective RF pulses to excite and refocus the off-resonant water surrounding the labeled cells. The method was designed to extend a sliceselection scheme to excite and refocus a thin 'shell' of spins 
away from the magnetized cells with a particular frequency offset. The resulting positive-contrast signal from the volume of cells is affected by the shape of the excitation profile, the center-frequency shift, and the echo-time. Therefore, extra scan procedures are required to find the optimal RF bandwidth and profile. In addition, an inherent limitation of the method is the complication associated with unwanted magnetization from the regions that suffer from chemical shift or susceptibility-related artifacts. Another method for positive-contrast imaging is achieved by using 'whitemarker' imaging by dephasing of the background signal by adjusting a slice-select gradient, and conserving the dipole field around the SPIO-labeled cells [4]. Since the positivecontrast is dependent on the amount of rephasing gradients (after the dephasing), the procedure may require optimization with respect to the iron content, field strength, echotime, and voxel resolution.

The methods mentioned above require a certain degree of parameter optimization and the implementation of special pulse sequences. Therefore, we were motivated to investigate a simple method to detect SPIO-labeled cells with positive-contrast using susceptibility weighted echo-time encoding technique (SWEET) [5].

\section{MATERIALS AND METHOD}

\section{A. Positive-contrast and SWEET}

The susceptibility measurement for the SPIO-labeled cells uses the susceptibility weighted echo-time encoding technique (SWEET) which enables the extraction of the susceptibility effect alone in MR imaging whereby the chemical shift and main field magnetic-field inhomogeneity effects are intermingled with the susceptibility effect of interest.

The implementation of SWEET sequence involved a simple modification of a conventional spin-echo sequence to increment an echo-time encoding step without adjusting the data acquisition windows [5]. In order to generate positive-contrast at the cell position, we acquired two sets of image volumes - one with a structural spin-echo image as a reference and the other with a susceptibility-weighted image with the echo-time shift.

\section{B. Cell preparation}

Human cervix carcinoma (KB) cells were used for labeling and imaging. First, KB cells $\left(20,000\right.$ cells $\left./ \mathrm{cm}^{2}\right)$ were cultured in $30 \mathrm{ml}$ of $10 \%$ fetal bovine serum (FBS) RPMI (Roswell Park Memorial Institute) medium (supplemented with $100 \mathrm{U} / \mathrm{ml}$ of penicillin and streptomycin) at $37^{\circ} \mathrm{C}$. Cell labeling solution was prepared using 750ug of the SPIObased dextran coated contrast agent ferumoxides (Feridex, Berlex laboratories Inc. Wayne, NJ), 11.25ug of poly-Llysine (PLL, Sigma, St. Louis, MO - as transfecting agent) and $15 \mathrm{ml}$ of serum-free RPMI medium. The cells were added to the labeling solution, and incubated for 1 hour at $37^{\circ} \mathrm{C}$ to allow for uptake of the particles. The RPMI medium was replaced with $15 \mathrm{ml}$ of the Feridex-PLL mixture and $15 \mathrm{ml}$ of serum-free RPMI medium, followed by incubation for 1 day at $37^{\circ} \mathrm{C}$. To remove the residual extracellular SPIO or PLL, the cells were washed three times with fresh phosphate-buffered saline (PBS).

\section{In-vitro gel phantom}

Gelatin phantoms were constructed using the $35 \mathrm{~mm}$ diameter vials. $10 \%(\mathrm{w} / \mathrm{v})$ gelatin solution was sterilized by autoclave. $30 \mathrm{ml}$ of gelatin solution pre-incubated at $37^{\circ} \mathrm{C}$ was poured into each vial. In order to place the group of cells in the middle of the well space, a microtube was inserted into the well containing the gelatin, and the microtube-vial ensemble was left to cool slowly at room temperature for 12 hours. During this time, the KB cells labeled with SPIO were isolated in the form of a pellet, mixed with small volume of the gelatin solution prepared at $37^{\circ} \mathrm{C}$. After the outer gelatin became fixated, the microtube was carefully redrawn from the gel, leaving tube-shaped gelatin 'groove' positioned at top-middle portion of the gelatin surface. The cell-gelatin mixture ( 10 ul in volume) was then poured in the groove. After the fixation, the solutionphase gelatin was carefully poured onto the ensemble to seal the labeled cell-pellet. The phantom was cooled slowly for 12 hours at room temperature to avoid trapping air bubbles inside. Phantoms containing seven different concentrations of cells- $1 \times 10^{6}, 5 \times 10^{5}, 2 \times 10^{5}, 1 \times 10^{5}, 5 \times 10^{4}, 1 \times 10^{4}$, and $5 \times 10^{3}$ cells- were prepared to help examine the visualization of a varied concentration of SPIO-labeled cells within a fixed area. The control sample was also prepared with $5 \times 10^{5}$ unlabeled KB cells.

\section{MR imaging}

All of MR imaging for this study were performed on a 3.0 T MRI system (ISOL, Korea) using a customized surface coil. SWEET sequence was implemented by simple modification of conventional spin-echo pulse sequence, capable of creating differential echo-time encoding steps (dT) for inducing the susceptibility weighted effect. All images were oriented trans-axially to depict the field inhomogeneity around the labeled cells and obtained with the following acquisition parameters; TR 500 ms, FOV 80×80 
$\mathrm{mm}^{2}$, matrix $256 \times 256$, flip angle $30^{\circ}$, slice thickness $2 \mathrm{~mm}$ (no gap), number of excitations (NEX) 2, number of slice 5, achieving the voxel size of $0.31 \times 0.31 \times 2 \mathrm{~mm}^{3}$. We acquired a conventional spin-echo image as an anatomical reference with TE $=40 \mathrm{~ms}$. To examine the effect of echo-time encoding variation, we adjusted the timing of 180 pulse to have six incremental echo timing ( $\mathrm{dT}=22.4,17.92,13.44$, 8.96, 4.48, $2.24 \mathrm{~ms}$ from the original TE). To confirm the location of the cell location, we acquired conventional gradient-echo images at several TEs (22.4, 17.92, 13.44, 8.96 $\mathrm{ms})$, covering the same imaging location.

\section{RESUlT}

The scan time was less than 5 minutes for each encoding step. Fig. 1 shows three consecutive slices (a, b, c) from the conventional spin-echo sequence. In each panel, upper left (i) contained a gelatin phantom including $5 \times 10^{5}$ unlabeled $\mathrm{KB}$ cells as a control; upper right (ii) contained $2 \times 10^{5}$, bottom left (iii) contained $5 \times 10^{5}$, and bottom right (iv) contained $1 \times 10^{6}$ labeled cells respectively.

Fig. 2 shows corresponding susceptibility weighted images by SWEET at echo-time encoding step (dT) of $22.4 \mathrm{~ms}$. In each image, the dark signal voids with four different numbers of Feridex-labeled cells were clearly visualized. Fig. 3 illustrates the positive-contrast images that were generated from difference of Fig. 1 and Fig. 2 (Fig. 1 minus Fig. 2). Effective removal of the background signal was clearly demonstrated. As the number of labeled KB cells increased, the areas showing the positive-contrast increased as well. Gradient-echo images acquired at $\mathrm{TE}=22.4 \mathrm{~ms}$ equivalent to one of the echo-time encoding steps of SWEET also demonstrated the variation of the susceptibility effect, comparable to SWEET method (shown in Fig. 4A Fig. 4B).
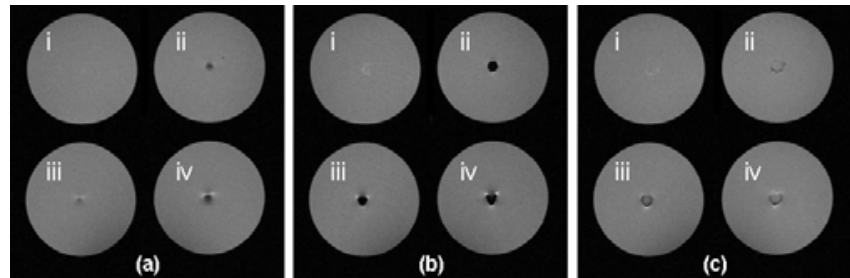

Fig. 1 Spin-echo images ( i: $5 \times 10^{5}$ unlabeled cells(control), ii: $2 \times 10^{5}$, iii: $5 \times 10^{5}$, iv: $1 \times 10^{6}$ Fe-labeled cells )
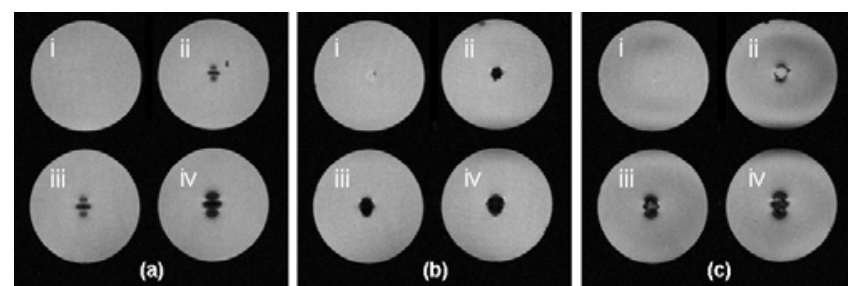

Fig. 2 SWEET images
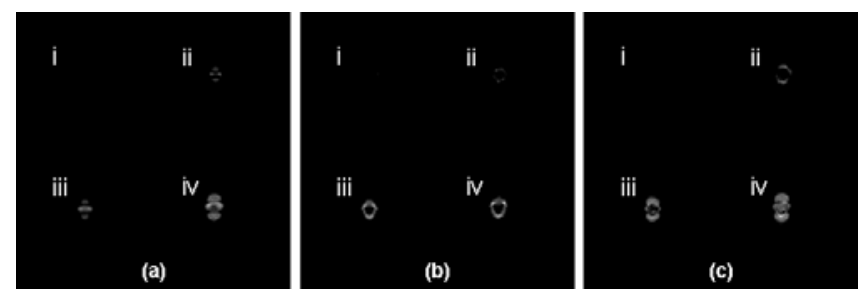

Fig. 3 Positive-contrast images
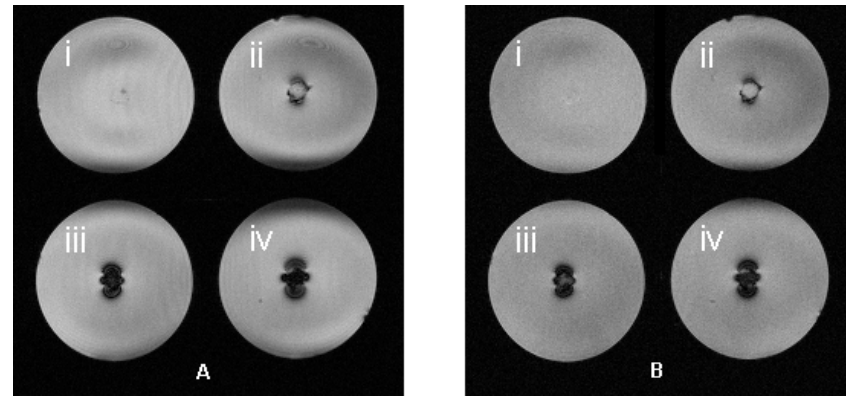

Fig. 4 (A) Gradient-echo images, (B) SWEET images with four different cell concentrations

Fig. 5A shows the number of voxels that are above the specific intensity threshold (above $10 \%$ of maximum signal intensity) with respect to the number of loaded cells. Fig. $5 \mathrm{~B}$, on the other hand, illustrates the averaging of the signal intensity from these voxels.

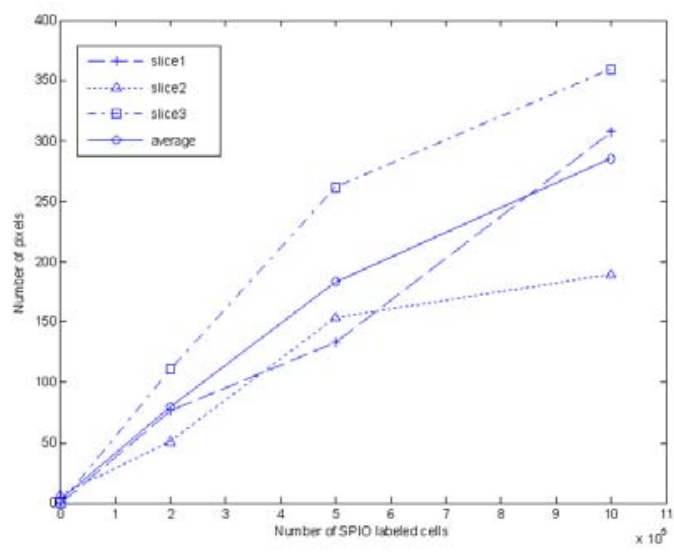

Fig. 5 (A) Number of pixels of positive-contrast in each slice 


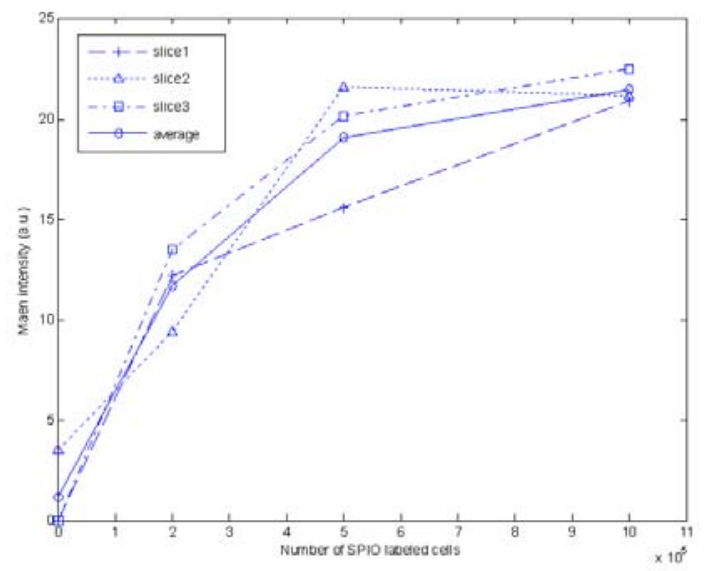

Fig. 5 (B) Mean intensity of positive-contrast in each slice

\section{Discussion}

We demonstrated the proposed method using SWEET could selectively enhance the effect of the field inhomogeneity caused by SPIO-labeled KB cells as positive-contrast. In Fig. 2, the signal reducing pattern was similar to the patterns observed from other studies [6, 7]. The number of positive pixels as well as the mean intensity increased proportionally with the number of labeled cells, however, at the high cell concentration (above $5 \times 10^{5}$ ), the effects started to taper off, indicating the saturation of the susceptibility effect.

Our method does not require the use of spatial-spectral pulses as in case of off-resonance imaging [3] with just a simple modification of a conventional spin-echo sequence. Most of the current cellular MRI techniques are based on the acquisition of gradient-echo images to detect the susceptibility caused by SPIO-labeled cells. However, the gradient-based method suffers from image distortion and artifacts, especially when a long TE is adopted to maximize the susceptibility effects. The SWEET approach, based on the spin-echo formation, is less vulnerable to these problems by separating the magnetic susceptibility effect from main field inhomogeneity and chemical shift artifacts. In addition, inner-volume imaging approaches via spatially-selective encoding schemes [8] are only available via spin-echo sequence family.

The limitation of the proposed method is that it requires at least two data acquisitions to generate the desired positive-contrast. However, we believe that it would not affect the overall scan time since reference images without echo shifting can be used to provide structural/anatomical information.

The high-resolution requirements of cellular MRI demands a high SNR (as well as CNR), therefore the adoption of fast and parallel imaging approaches can be gainfully applied to our proposed method to increase the SNR necessary for cell detection. The examination of the efficacy of our method in an animal model as well as MRI monitoring of the proliferation of cancer cells constitutes the direction of our future investigation.

\section{ACKNOWLEDGMENT}

This work is supported by Korean Ministry of Commerce, Industry and Energy (MOCIE), Grant \# 2004-02012.

\section{REFERENCES}

1. K. C. Wolbert, H. Drexler (2006) Cell-based therapy for heart failure. Curr. Oppin. Radiol. 3;234-239.

2. Charles H. Cunningham, Takayasu Arai, Philip C. Yang, Michel V. McConnell, John M. Pauly, and Steven M. Conolly (2005) Positive contrast magnetic resonance imaging of cells labeled with magnetic nanoparticles. Magnet Reson Med 53:999-1005

3. M. Stuber, W. D. Gilson, M. Schaer, J. W. Bulte, D. L. Kraitchman (2005) Shedding light on the dark spot with IRON - a method that generated positive contrast in the presence of superparamagnetic nanoparticles. Proc Intl. Soc. Mag. Reson. Med. Miami, USA, p.2608

4. Venkatesh Mani, Karen C. Briley-Saebo, Vitalii V. Itskovich, Daniel D. Samber, Zahi A. Fayad (2006) Gradient echo acquisition for superparamagnetic particles with positive contrast (GRASP): sequence characterization in membrane and glass superparamagnetic iron oxide phantoms at 1.5T and 3T. Magnet Reson Med 55:126-135

5. HW Park, YM Ro, ZH Cho (1988) Measurement of the magnetic susceptibility effect in high-field NMR imaging. Phys. Med. Biol. 33(3):339-349

6. Jan-Henry Seppenwoolde, Max A. Viergever, Chris J.G. Bakker (2003) Passive tracking exploiting local signal conservation: The white marker phenomenon. Magnet Reson Med 503:784-790

7. Chris Heyn, Chris V. Bowen, Brian K. Rutt, Paula J. Foster (2005) Detection threshold of single SPIO-labeled cells with FIESTA. Magnet Reson Med 53:312-320

8. D. Mitsouras, R.V. Mulkern, F. J. Rybicki (2006) Strategies for inner volume 3D fast spin echo magnetic resonance imaging using nonselective refocusing radio frequency pulses. Med. Phys. 33(1): 173-186

Address of the corresponding author:

Author: HyunWook Park

Institute: Korea Advanced Institute of Science and Technology (KAIST)

Street: $\quad 373-1$ Guseong-dong, Yuseong-gu

City: Daejeon

Country: Korea

Email: hwpark@athena.kaist.ac.kr 